\title{
Precise predictions for the Higgs production in association with a W-boson pair at ILC
}

\author{
Song Mao, Ma Wen-Gan, Zhang Ren-You, Guo Lei, and Wang Shao-Ming \\ Department of Modern Physics, University of Science and Technology \\ of China (USTC), Hefei, Anhui 230027, P.R.China
}

\begin{abstract}
The Higgs-boson production in association with a W-boson pair at $e^{+} e^{-}$linear colliders is one of the important processes in probing the coupling between Higgsboson and vector gauge bosons and discovering the signature of new physics. We describe the impact of the complete electroweak $(\mathrm{EW})$ radiative corrections of $\mathcal{O}\left(\alpha_{\text {ew }}\right)$ to this process in the standard model(SM) at the International Linear Collider(ILC), and investigate the dependence of the lowest-order(LO) and EW next-to-leading or$\operatorname{der}(\mathrm{NLO})$ corrected cross sections on colliding energy $\sqrt{s}$ and Higg-boson mass. The LO and NLO EW corrected distributions of the invariant mass of W-boson pair and the transverse momenta of final $W$ - and Higgs-boson are presented. Our numerical results show that the relative $\mathrm{EW}$ radiative correction $\left(\delta_{\text {ew }}\right)$ varies from $-19.4 \%$ to $0.2 \%$ when $m_{H}=120 \mathrm{GeV}$ and $\sqrt{s}$ goes up from $300 \mathrm{GeV}$ to $1.2 \mathrm{TeV}$.
\end{abstract}

Keywords: Higgs-boson production, W-boson pair, electroweak interaction, radiative correction

PACS: 12.15.Lk, 14.80.Bn, 14.70.Fm, 11.80.Fv 


\section{Introduction}

The Higgs-boson plays a very important role in the standard model(SM), it is responsible for the breaking of the electroweak(EW) symmetry and the generation of masses for the fundamental particles [1, 2]. Unfortunately, it has not been directly detected yet in experiments. Searching for Higgs-boson within the SM and studying the phenomenology concerning its properties are the important tasks for the present and upcoming high energy colliders. Until now, we have obtained a lot of experimental data which agree with the predictions of the SM. These precise data constrain strongly the couplings of the gauge-boson to fermions and the couplings between vector gauge bosons, but present little information about the couplings between Higgs-boson and gauge bosons. Previous LEP experiments have provided the lower limit on the SM Higgs-boson mass as $114.4 \mathrm{GeV}$ at the $95 \%$ confidence level, which is extracted from the results of searches for $e^{+} e^{-} \rightarrow Z^{0} H^{0}$ production [3, 4]. The present indirect evidences of the SM Higgs-boson mass through EW

precision measurements indicate the $95 \%$ C.L. upper bound as $m_{H} \lesssim 182 \mathrm{GeV}$, when the lower limit on $m_{H}$ is used in determination of this upper limit [4].

Compared to hadron machine, $e^{+} e^{-}$linear collider has the advantage of producing Higgs-boson in a particularly clean environment. That is essential for studying Higgsboson properties since the cross section of Higgs-boson production is rather small due to the fact that the scalar Higgs-boson couples mainly to heavy particles. Furthermore, in the upper and lower limits for the SM Higgs-boson mass, $H^{0}$ decays mainly into the bottom-quark pair allowed by phase-space. Therefore, the properties of Higgs-boson with the mass in present limitation range, are very hard to be precisely probed at hadron colliders because of huge QCD backgrounds.

The future International Linear Collider (ILC) is designed as a machine with the entire colliding energy in a range of $200 \mathrm{GeV}<\sqrt{s}<500 \mathrm{GeV}$ and an integrated luminosity 
of around $500(f b)^{-1}$ in four years. The machine could be upgraded to $\sqrt{s} \sim 1 \mathrm{TeV}$ with an integrated luminosity of $1(a b)^{-1}$ in three years [5]. At this machine, Higgsboson would be produced mainly via the Higgs-boson strahlung process, and the coupling of Higgs-boson to $Z$-bosons is probed best in the measurement of the cross sections of the Higgs-boson strahlung process $e^{+} e^{-} \rightarrow H^{0} Z^{0}$ and the $W W / Z Z$ fusion processes $e^{+} e^{-} \rightarrow H^{0} \nu \bar{\nu}$ and $e^{+} e^{-} \rightarrow H^{0} e^{+} e^{-}$. The ILC would be an ideal machine to observe and study in detail an intermediate-mass or even a heavier Higgs-boson [6, 7]. It is sure that once the neutral Higgs-boson is discovered and its mass is determined, the $H V V$ production through $e^{+} e^{-} \rightarrow H^{0} V V(V=Z, W, \gamma)$ processes may provide the detail information of the coupling between Higgs-boson and vector gauge bosons, which directly reflects the role of the Higgs-boson in EW symmetry breaking. Moreover, a theoretical accurate estimation of these processes is essential, since $e^{+} e^{-} \rightarrow H^{0} V V(V=Z, W, \gamma)$ processes could be potential backgrounds for possible new physics. The investigation of the processes $e^{+} e^{-} \rightarrow H^{0} W^{+} W^{-}, e^{+} e^{-} \rightarrow H^{0} Z^{0} Z^{0}$ and $e^{+} e^{-} \rightarrow H^{0} Z^{0} \gamma$ for testing the couplings between Higgs-boson and gauge bosons were studied at the tree-level in Ref.[9, 10]. The coupling of $g_{H Z Z}$ can be determined at a few percent level for a $120 \mathrm{GeV}$ Higgs-boson with an integrated luminosity of $500 \mathrm{fb}^{-1}$ from the production cross section of the process $e^{+} e^{-} \rightarrow H^{0} Z^{0} Z^{0}$ as shown in Ref.[8]. Recently, Y.-J. Zhou et al., studied the full $\mathcal{O}\left(\alpha_{e w}\right)$ EW corrections to the process $e^{+} e^{-} \rightarrow H^{0} Z^{0} Z^{0}[11$. They conclude that the $\mathcal{O}\left(\alpha_{\text {ew }}\right)$ EW radiative corrections to $e^{+} e^{-} \rightarrow H^{0} Z^{0} Z^{0}$ process are in the range between $-15 \%$ and $1.0 \%$ when $\sqrt{s}$ varies from $400 \mathrm{GeV}$ to $2 \mathrm{TeV}$, which should be taken into consideration in the future precise experiments. The process of Higgs-boson production in association with a pair of W-bosons at the ILC is another significant process. It is not only important in probing $g_{H W W}$ coupling, but also possible to provide further tests for the quadrilinear couplings, such as $H W W Z$ and $H W W \gamma$, which do not exist at treelevel in the SM, because these quadrilinear couplings would induce deviations from the 
SM predicted observables. That means the precise theoretical prediction is necessary in experimental data analysis for $e^{+} e^{-} \rightarrow H^{0} W^{+} W^{-}$process.

In this work we calculate the full one-loop EW corrections to the process $e^{+} e^{-} \rightarrow$ $H^{0} W^{+} W^{-}$in the SM. The paper is arranged as follows: In Section II we give the calculation description of the Born cross section, and the calculation of full $\mathcal{O}\left(\alpha_{e w}^{4}\right)$ EW radiative contribution to the $e^{+} e^{-} \rightarrow H^{0} W^{+} W^{-}$process is provided in section III. In Section IV we present some numerical results and discussion, and finally a short summary is given.

\section{LO contributions of $\mathcal{O}\left(\alpha_{e w}^{3}\right)$ to $e^{+} e^{-} \rightarrow H^{0} W^{+} W^{-}$ process}

In our LO and NLO calculations we apply FeynArts3.3 package[12] to generate Feynman diagrams and convert them to corresponding amplitudes. The amplitude reductions are mainly implemented by applying FormCalc5.3 programs[13]. We use the 't HooftFeynman gauge in the leading-order calculation, if there is no other statement. The contribution to the process $e^{+} e^{-} \rightarrow H^{0} W^{+} W^{-}$at the order of $\mathcal{O}\left(\alpha_{e w}^{3}\right)$ in the $\mathrm{SM}$ is based on pure electroweak interactions. We ignore the contribution from the Feynman diagrams which involve electron-Higgs Yukawa coupling, because the concerning Yukawa coupling strength is proportional to the electron mass, and the electron mass is negligible comparing with the colliding energy $\sqrt{s}$ at the ILC, Then there are eleven Feynman diagrams which we consider in the calculation for the process $e^{+} e^{-} \rightarrow H^{0} W^{+} W^{-}$at the tree-level(shown in Fig,1). The Feynman diagrams in Fig,1 topologically can be divided into s-channel diagrams with intermediate $\gamma$ - or $Z^{0}$-boson, and t-channel diagrams with $\nu_{e}$-exchanging. The notations for the process $e^{+} e^{-} \rightarrow H^{0} W^{+} W^{-}$are defined as

$$
e^{+}\left(p_{1}\right)+e^{-}\left(p_{2}\right) \rightarrow H^{0}\left(p_{3}\right)+W^{+}\left(p_{4}\right)+W^{-}\left(p_{5}\right),
$$




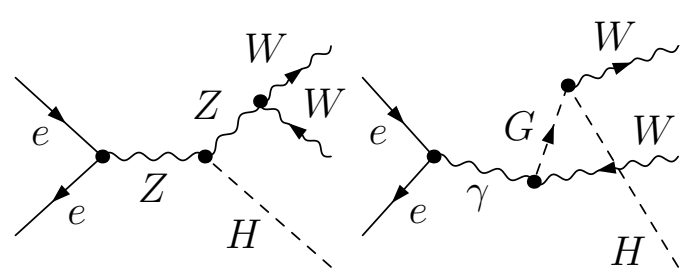

(1)

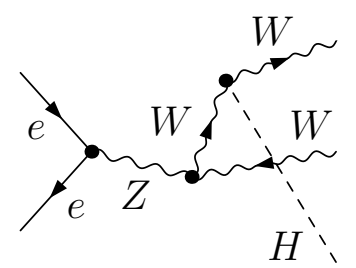

(5)

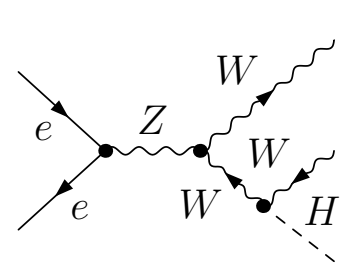

(9)
$(2)$

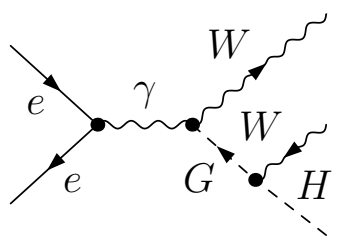

(6)

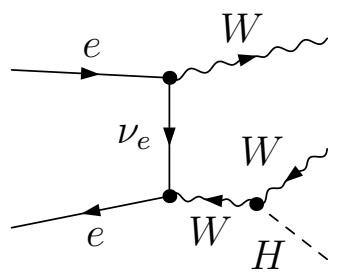

(10)

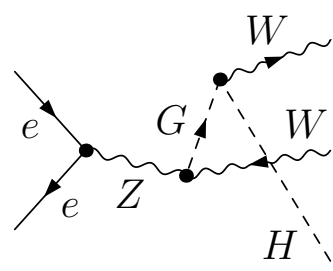

(3)

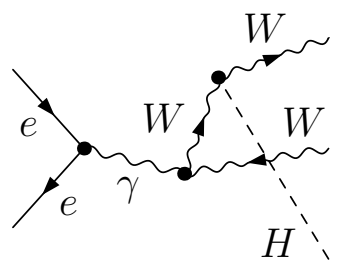

(4)

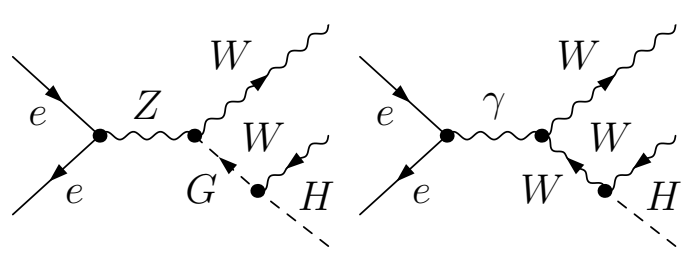

(8)

$(7)$

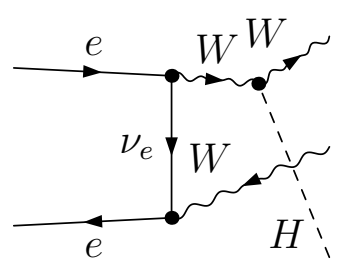

(11)

Figure 1: The full set of tree-level Feynman diagrams for the $e^{+} e^{-} \rightarrow H^{0} W^{+} W^{-}$process neglecting the electron-Higgs coupling.

where $p_{i}(i=1-5)$ label the four-momenta of incoming positron, electron and outgoing particles, respectively. The differential cross section for the process $e^{+} e^{-} \rightarrow H^{0} W^{+} W^{-}$ at the tree-level is then obtained as

$$
d \sigma_{\text {tree }}=\frac{1}{4} \sum_{\text {spin }}\left|\mathcal{M}_{\text {tree }}\right|^{2} d \Phi_{3},
$$

where $\mathcal{M}_{\text {tree }}$ is the amplitude of all the tree-level diagrams shown in Fig 1. The factor $\frac{1}{4}$ is due to taking average over the spins of the initial particles. $d \Phi_{3}$ is the three-particle phase space element defined as

$$
d \Phi_{3}=\delta^{(4)}\left(p_{1}+p_{2}-\sum_{i=3}^{5} p_{i}\right) \prod_{j=3}^{5} \frac{d^{3} \boldsymbol{p}_{j}}{(2 \pi)^{3} 2 E_{j}} .
$$




\section{Virtual and real photon emission corrections to $e^{+} e^{-} \rightarrow H^{0} W^{+} W^{-}$process}

The one-loop Feynman diagrams and relevant counterterm diagrams of process $e^{+} e^{-} \rightarrow$ $H^{0} W^{+} W^{-}$are also generated by using FeynArts3.3 package. At the EW NLO, there are as many as 2770 one-loop Feynman diagrams being taken into account in our calculation, and they can be classified into self-energy, triangle(3-point), box(4-point), pentagon(5point) and counterterm diagrams. There are 82 pentagon diagrams. A representative set of pentagon diagrams can be found in Fig, 2. The calculation of the one-loop diagrams has been performed in the conventional 't Hooft-Feynman gauge. The virtual contribution of $\mathcal{O}\left(\alpha_{e w}^{4}\right)$ to $e^{+} e^{-} \rightarrow H^{0} W^{+} W^{-}$process can be expressed as [14]

$$
\Delta \sigma_{\text {virtual }}=\sigma_{\text {tree }} \delta_{\text {virtual }}=\frac{(2 \pi)^{4}}{2\left|\vec{p}_{1}\right| \sqrt{s}} \int \frac{1}{4} \mathrm{~d} \Phi_{3} \sum_{\text {spin }} \operatorname{Re}\left(\mathcal{M}_{\text {tree }} \mathcal{M}_{\text {virtual }}^{\dagger}\right),
$$

where $\vec{p}_{1}$ is the c.m.s. spatial momentum of the incoming positron. $\mathcal{M}_{\text {virtual }}$ represents the amplitude of $\mathcal{O}\left(\alpha_{e w}^{4}\right)$ order Feynman diagrams including EW one-loop and counterterm diagrams. We use the dimensional regularization(DR) method to isolate the ultraviolet(UV) and infrared(IR) singularities. The numerical calculation of the one-, two-, threeand four-point integral functions are implemented by using the expressions presented in Refs. [15, 16]. The implementations of the scalar, vector and tensor five-point integrals are done exactly by using the approach presented in Refs. [17, 18]. The $\mathcal{O}\left(\alpha_{e w}^{4}\right)$ calculation of the cross section for hard photon radiation process $e^{+} e^{-} \rightarrow H^{0} W^{+} W^{-} \gamma$ is accomplished by using Grace2.2.1 package[19].

The total unrenormalized amplitude corresponding to all the one-loop Feynman diagrams contains both ultraviolet (UV) and infrared (IR) divergences. The UV and IR divergences in loop integrals are isolated by adopting the dimensional regularization(DR) scheme. The relevant fields are renormalized by taking the on-mass-shell (OMS) scheme [20]. As we expect, the UV divergence contributed by virtual one-loop diagrams can 


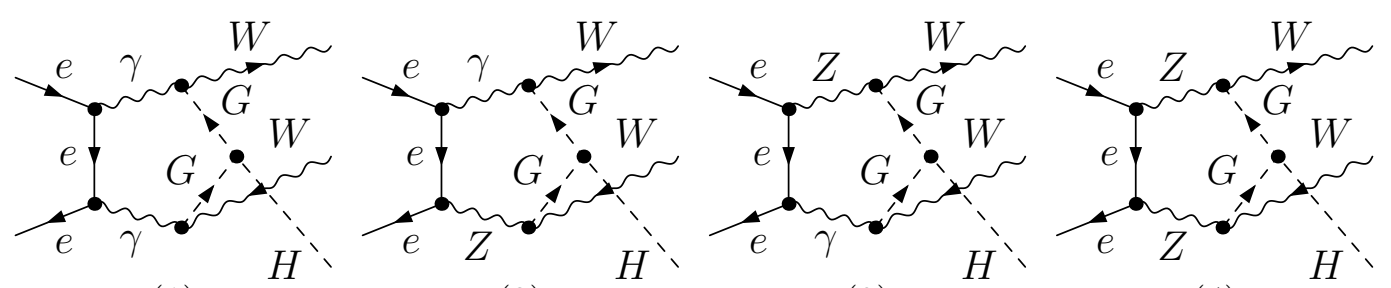

(1)

(2)

(3)

(4)

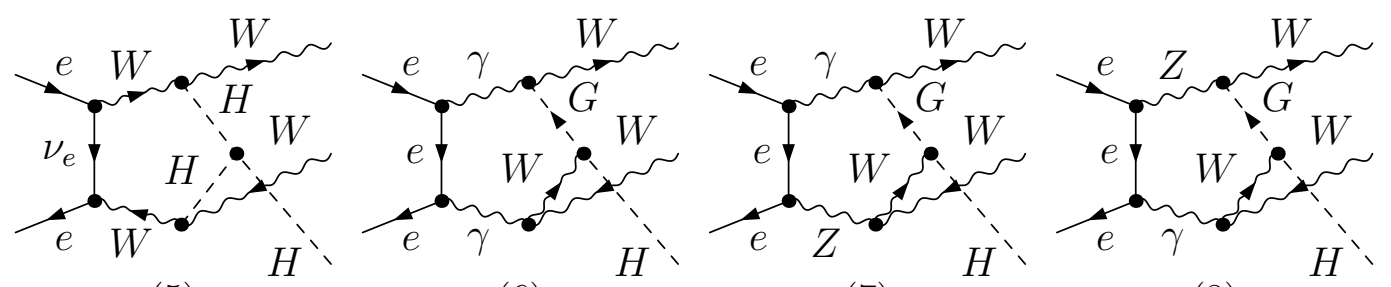

(5)

(6)

(7)

(8)

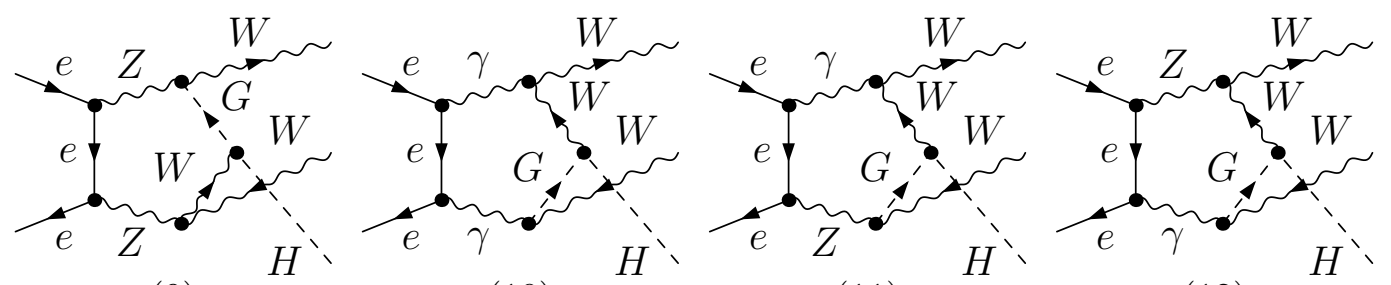

(9)

(10)

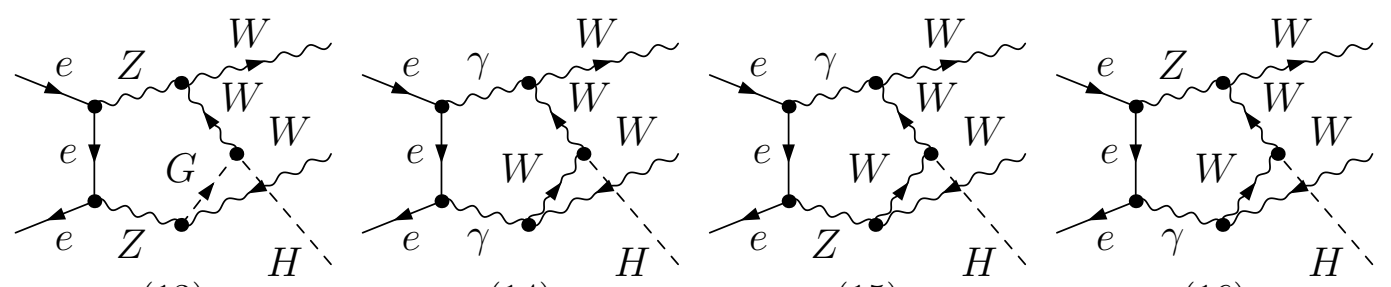

(13)

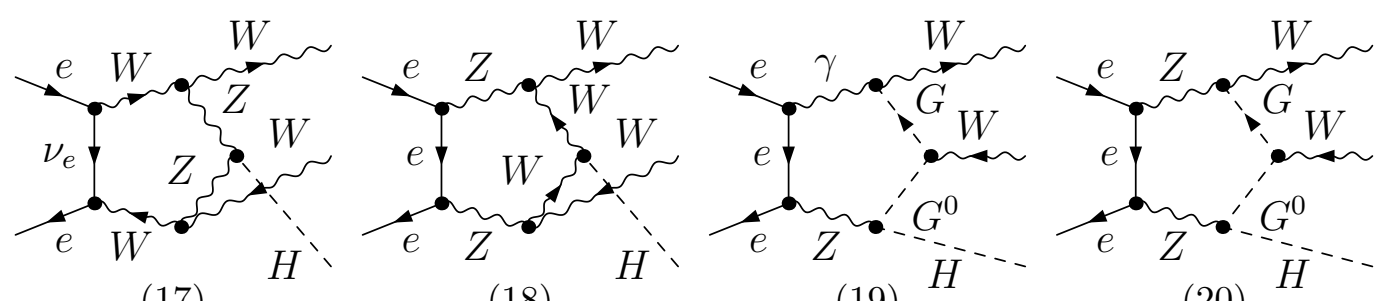

(17)

Figure 2: Some representative pentagon Feynman diagrams for the process $e^{+} e^{-} \rightarrow$ $H^{0} W^{+} W^{-}$. 
be cancelled by that contributed from the counterterms exactly both analytically and numerically.

In our one-loop calculation there exists soft IR divergence, but no collinear IR singularity, because we keep nonzero electron(positron) mass. The soft IR divergence in virtual photonic corrections for the process $e^{+} e^{-} \rightarrow H^{0} W^{+} W^{-}$can be exactly cancelled by adding the real photonic bremsstrahlung corrections to this process in the soft photon limit. In the real photon emission process, denoted as

$$
e^{+}\left(p_{1}\right)+e^{-}\left(p_{2}\right) \rightarrow H^{0}\left(p_{3}\right)+W^{+}\left(p_{4}\right)+W^{-}\left(p_{5}\right)+\gamma\left(p_{6}\right),
$$

a real photon radiates from the electron/positron or W-bosons, and can be soft or hard. We adopt the general phase-space-slicing (PSS) method [21] to isolate the soft photon emission singularity part in the real photon emission process $e^{+} e^{-} \rightarrow H^{0} W^{+} W^{-} \gamma$. The bremsstrahlung phase space is divided into singular and non-singular regions, and the cross section of the $e^{+} e^{-} \rightarrow H^{0} W^{+} W^{-} \gamma$ process is decomposed into soft and hard terms,

$$
\Delta \sigma_{\text {real }}=\Delta \sigma_{\text {soft }}+\Delta \sigma_{\text {hard }}=\sigma_{\text {tree }}\left(\delta_{\text {soft }}+\delta_{\text {hard }}\right) .
$$

We can consider that the radiated photon in the reaction $e^{+} e^{-} \rightarrow H^{0} W^{+} W^{-} \gamma$ can be either soft or hard, depending on the photon energy $\left(E_{6}\right)$ in the center of mass system(c.m.s.) frame. The criterion is like that: if $E_{6} \leq \Delta E$, the radiated photon is soft, otherwise it is hard, where $\Delta E$ is defined as $\Delta E \equiv \delta_{s} E_{b}$, and $E_{b}$ is the electron beam energy in the c.m.s. frame and equals to $\sqrt{s} / 2$. Then theoretically both $\Delta \sigma_{\text {soft }}$ and $\Delta \sigma_{\text {hard }}$ should depend on the arbitrary soft cutoff $\delta_{s}$, but the total EW one-loop correction $\left(\Delta \sigma_{\text {tot }}\right)$ and $\Delta \sigma_{\text {real }}$ should be cutoff $\delta_{s}$ independent. If the IR singularity in the soft photon emission process is cancelled exactly with that from the virtual photonic corrections, the independence of $\Delta \sigma_{\text {tot }}\left(\equiv \Delta \sigma_{\text {virtual }}+\Delta \sigma_{\text {real }}\right)$ on the cutoff $\delta_{s}$ and fictitious photon mass $m_{\gamma}$, could be demonstrated in our calculculation. Since generally we take the soft cutoff $\delta_{s}$ to be a small value during our calculations, the terms of order $\Delta E / E_{b}$ can be neglected and the 
soft contribution can be evaluated by using the soft photon approximation analytically [20, 22

$$
\mathrm{d} \Delta \sigma_{\text {soft }}=-\mathrm{d} \sigma_{\text {tree }} \frac{\alpha_{\mathrm{ew}}}{2 \pi^{2}} \int_{\left|\vec{p}_{6}\right| \leq \Delta E} \frac{\mathrm{d}^{3} \vec{p}_{6}}{2 p_{6}^{0}}\left[\frac{p_{1}}{p_{1} \cdot p_{6}}-\frac{p_{2}}{p_{2} \cdot p_{6}}-\frac{p_{4}}{p_{4} \cdot p_{6}}+\frac{p_{5}}{p_{5} \cdot p_{6}}\right]^{2},
$$

where we define the four-momentum of radiated photon as $p_{6}=\left(p_{6}^{0}, \vec{p}_{6}\right)$. As shown in Eq.(3.4), the soft contribution has an IR singularity at $\Delta E=0$, which can be cancelled exactly with that from the virtual photonic corrections. The hard contribution is UV and IR finite, and can be computed directly by using the Monte Carlo technique. Finally, the corrected total cross $\operatorname{section}\left(\sigma_{\text {tot }}\right)$ up to the order of $\mathcal{O}\left(\alpha_{\text {ew }}^{4}\right)$ for the $e^{+} e^{-} \rightarrow H^{0} W^{+} W^{-}$ process is obtained by summing the $\mathcal{O}\left(\alpha_{e w}^{3}\right)$ Born cross $\operatorname{section}\left(\sigma_{\text {tree }}\right)$, the $\mathcal{O}\left(\alpha_{e w}^{4}\right)$ virtual $\operatorname{cross} \operatorname{section}\left(\Delta \sigma_{\text {virtual }}\right)$, and the $\mathcal{O}\left(\alpha_{e w}^{4}\right)$ cross section of the real photon emission process $e^{+} e^{-} \rightarrow H^{0} W^{+} W^{-} \gamma\left(\Delta \sigma_{\text {real }}\right)$.

$$
\sigma_{\text {tot }}=\sigma_{\text {tree }}+\Delta \sigma_{\text {tot }}=\sigma_{\text {tree }}+\Delta \sigma_{\text {virtual }}+\Delta \sigma_{\text {real }}=\sigma_{\text {tree }}\left(1+\delta_{\text {ew }}\right),
$$

where $\delta_{\text {ew }}=\delta_{\text {virtual }}+\delta_{\text {soft }}+\delta_{\text {hard }}$ is the full $\mathcal{O}\left(\alpha_{\text {ew }}\right)$ electroweak relative correction.

\section{Numerical results and discussion}

For the numerically verification of the gauge invariance in our LO calculation, we used Grace2.2.1 and FeynArts3.3/FormCalc5.3 packages separately, and adopted 't HooftFeynman gauge and unitary gauge respectively in calculating the Born cross section of the process $e^{+} e^{-} \rightarrow H^{0} W^{+} W^{-}$, and got the coincident numerical results. We use also both Grace2.2.1 and FeynArts3.3/FormCalc5.3 packages to calculate the tree-level cross section of process $e^{+} e^{-} \rightarrow H^{0} W^{+} W^{-}$in 't Hooft-Feynman gauge with the input parameters used in Ref.[10], and compare our numerical results with those in Ref.[10] in order to verify again the correctness of our tree-level calculation. The input parameters used in Ref.[10] are taken as: $m_{Z}=91.18 \mathrm{GeV}, m_{W}=80.1 \mathrm{GeV}, \alpha_{e w}\left(m_{Z}\right)=1 / 128, \sqrt{s}=500 \mathrm{GeV}$ and 


\begin{tabular}{|c|c|c|c|}
\hline$m_{H}(\mathrm{GeV})$ & $\sigma_{\text {tree }}(\mathrm{fb})($ Ref.[10] $)$ & $\sigma_{\text {tree }}(\mathrm{fb})($ Grace $)$ & $\sigma_{\text {tree }}(\mathrm{fb})($ FeynArts $)$ \\
\hline 120 & 5.63 & $5.6378(6)$ & $5.6374(6)$ \\
\hline 150 & 3.51 & $3.5123(3)$ & $3.5126(3)$ \\
\hline 200 & 1.47 & $1.4714(1)$ & $1.4715(1)$ \\
\hline
\end{tabular}

Table 1: The comparison of the numerical results of the tree-level cross sections for the process $e^{+} e^{-} \rightarrow H^{0} W^{+} W^{-}$with the corresponding ones in Ref. [10], by taking the same input parameters as in Ref.[10] and using Grace2.2.1 and FeynArts3.3/FormCalc5.3 packages separately.

an effective $\sin ^{2} \theta_{W}=0.232$. The numerical results of the tree-level cross section for the process $e^{+} e^{-} \rightarrow H^{0} W^{+} W^{-}$are listed in Table 1. We can see that Table 11 yields results in mutual agreement between ours and those presented in Ref.[10].

The following numerical computation is performed in the $\alpha_{e w}$-scheme and the initial EW input physical parameters are taken as [14]:

$$
m_{W}=80.403 \mathrm{GeV}, \quad m_{Z}=91.1876 \mathrm{GeV}, \quad \alpha_{\text {ew }}(0)=1 / 137.03599911 .
$$

The charged leptons have the mass values as

$$
m_{e}=0.51099892 \mathrm{MeV}, m_{\mu}=105.658369 \mathrm{MeV}, m_{\tau}=1776.99 \mathrm{MeV}
$$

For the quark masses, beside the top-quark mass $m_{t}=172.5 \mathrm{GeV}$, we take the set $m_{u}=m_{d}=66 \mathrm{MeV}, m_{s}=150 \mathrm{MeV}, m_{c}=1.5 \mathrm{GeV}$, and $m_{b}=4.7 \mathrm{GeV}$. Since quark-mixing effects are suppressed, we set the CKM matrix to the unit matrix.

Except the input parameters shown above, we have to fix the values of the IR regulator $m_{\gamma}$, the fictitious photon mass, and soft cutoff $\delta_{s}=\Delta E / E_{b}$ during our one-loop numerical calculation. In fact, if the one-loop calculation is correct and the IR divergency is really cancelled, the total cross section should be independent of these two parameters. Our numerical results show that the cross section contribution at $\mathcal{O}\left(\alpha_{\text {ew }}^{4}\right)$ order, $\Delta \sigma_{\text {tot }}=$ $\Delta \sigma_{\text {real }}+\Delta \sigma_{\text {virtual }}$, is invariant within the calculation errors when the fictitious photon 

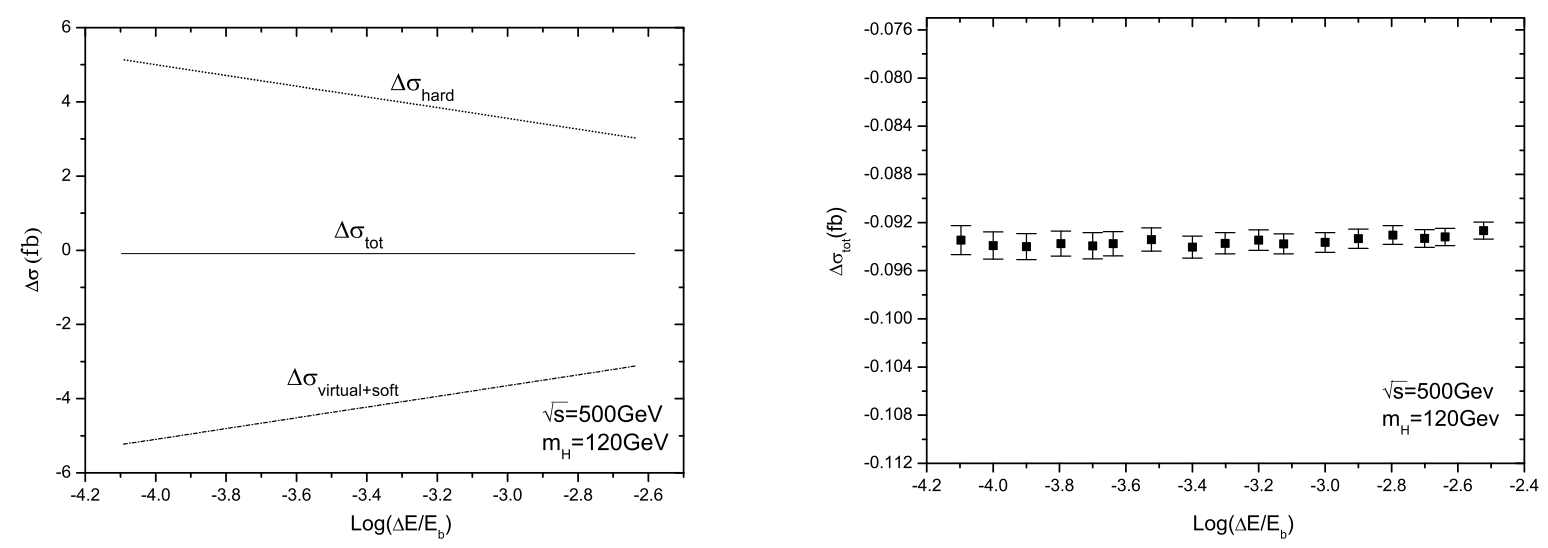

Figure 3: (a) The $\mathcal{O}\left(\alpha_{\text {ew }}^{4}\right)$ contribution parts of cross section for $e^{+} e^{-} \rightarrow H^{0} W^{+} W^{-}$ process as the functions of the soft cutoff $\delta_{s} \equiv \Delta E / E_{b}$ in conditions of $m_{\gamma}=10^{-10} \mathrm{GeV}$, $m_{H}=120 \mathrm{GeV}$ and $\sqrt{s}=500 \mathrm{GeV}$. (b) The amplified curve for $\Delta \sigma_{\text {tot }}$ of Fig.3(a) versus $\delta_{s}$ including calculation errors.

mass $m_{\gamma}$ varies from $10^{-15} \mathrm{GeV}$ to $10^{-1} \mathrm{GeV}$ in conditions of $\delta_{s}=10^{-3}, m_{H}=120 \mathrm{GeV}$ and $\sqrt{s}=500 \mathrm{GeV}$.

As a check of the correctness in our calculation of radiative correction, we present Figs $3(a-b)$ to show the independence of the total $\mathcal{O}\left(\alpha_{\text {ew }}^{4}\right)$ electroweak contribution to $e^{+} e^{-} \rightarrow H^{0} W^{+} W^{-}$process on soft cutoff $\delta_{s}$, assuming $m_{\gamma}=10^{-5} \mathrm{GeV}, m_{H}=120 \mathrm{GeV}$ and $\sqrt{s}=500 \mathrm{GeV}$. As shown in Fig[3(a), both $\Delta \sigma_{\text {virtual }}+\Delta \sigma_{\text {soft }}$ and $\Delta \sigma_{\text {hard }}$ strongly depend on soft cutoff $\delta_{s}$, but the total $\mathcal{O}\left(\alpha_{\text {ew }}^{4}\right)$ EW relative radiative contribution $\Delta \sigma_{\text {tot }}=$ $\Delta \sigma_{\text {virtual }}+\Delta \sigma_{\text {real }}$ is cutoff $\delta_{s}$ independent within the range of calculation errors as expected. In order to show the curve of $\Delta \sigma_{t o t}$ more clearly, we present the amplified curve for $\Delta \sigma_{t o t}$ including calculation errors in Fig, 3 (b). In further calculations, we fix $m_{\gamma}=10^{-5} \mathrm{GeV}$ and $\delta_{s}=10^{-3}$.

In Fig,4(a) we present the curves of the LO and one-loop EW corrected cross sections as the functions of colliding energy $\sqrt{s}$, with $m_{H}=120 \mathrm{GeV}, 150 \mathrm{GeV}, 200 \mathrm{GeV}$, separately. Fig 4 (b) shows their corresponding relative EW radiative corrections $\left(\delta_{e w} \equiv \frac{\Delta \sigma_{t o t}}{\sigma_{\text {tree }}}\right)$ for 

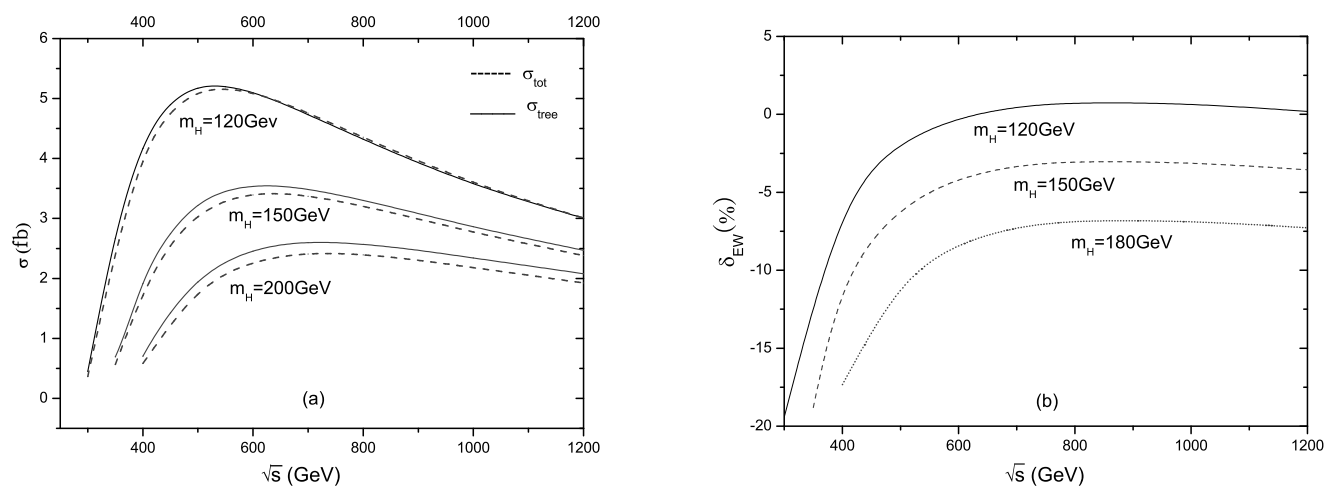

Figure 4: (a) The LO and EW one-loop corrected cross sections for the process $e^{+} e^{-} \rightarrow$ $H^{0} W^{+} W^{-}$as the functions of colliding energy $\sqrt{s}$ with $m_{H}=120 \mathrm{GeV}, 150 \mathrm{GeV}$, $180 \mathrm{GeV}$ respectively. (b) The corresponding relative EW radiative corrections $\left(\delta_{e w}\right)$ versus $\sqrt{s}$.

the data presented in Fig, $4(\mathrm{a})$. We find from Figs, $4(\mathrm{a}-\mathrm{b})$ that the LO cross sections are sensitive to the Higgs-boson mass and generally suppressed by the one-loop EW corrections except for $m_{H}=120 \mathrm{GeV}$ and the colliding energy being in the range of $\sqrt{s}>$ $630 \mathrm{GeV}$. Fig 4 (b) shows that the relative radiative correction due to full EW one-loop contributions in the $\sqrt{s}$ vicinity close to the threshold of $H^{0} W^{+} W^{-}$production, becomes rather large. That is because of the Coulomb singularity effect from the diagrams involving the instantaneous virtual photon exchange in loop which has a small spatial momentum. To show the numerical results as presented in Figs,4(a-b) more precisely, we list some typical numerical results of the tree-level, one-loop EW corrected cross sections and the relative EW radiative correction $\left(\delta_{e w} \equiv \Delta \sigma_{\text {tot }} / \sigma_{\text {tree }}\right)$ for the process $e^{+} e^{-} \rightarrow H^{0} W^{+} W^{-}$in Table 2. There they are in conditions of $\sqrt{s}=400 \mathrm{GeV}, 600 \mathrm{GeV}, 800 \mathrm{GeV}, 1000 \mathrm{GeV}$, $1200 \mathrm{GeV}$ and $m_{H}=120 \mathrm{GeV}, 150 \mathrm{GeV}, 180 \mathrm{GeV}$ separately. We can read out also from Fig $4(\mathrm{~b})$ that when $m_{H}=120 \mathrm{GeV}$ and $\sqrt{s}$ goes up from $300 \mathrm{GeV}$ to $1.2 \mathrm{TeV}$, the relative EW radiative correction, $\delta_{\text {ew }}$, increases from $-19.4 \%$ to $0.2 \%$.

In Fig $5(a)$ we present the plot of the LO and EW one-loop corrected cross sections 


\begin{tabular}{|c|c|c|c|c|}
\hline$\sqrt{s}(\mathrm{GeV})$ & $m_{H}(\mathrm{GeV})$ & $\sigma_{\text {tree }}(f b)$ & $\sigma_{\text {tot }}(f b)$ & $\delta_{\text {ew }}(\%)$ \\
\hline & 120 & $4.1676(4)$ & $3.953(2)$ & $-5.15(5)$ \\
400 & 150 & $1.9144(2)$ & $1.719(1)$ & $-10.21(5)$ \\
& 180 & $0.70438(7)$ & $0.5823(5)$ & $-17.33(7)$ \\
\hline \multirow{3}{*}{600} & 120 & $5.0945(5)$ & $5.085(3)$ & $-0.19(6)$ \\
& 150 & $3.5357(3)$ & $3.392(2)$ & $-4.06(6)$ \\
& 180 & $2.4562(3)$ & $2.254(2)$ & $-8.23(8)$ \\
\hline \multirow{3}{*}{800} & 120 & $4.3205(6)$ & $4.354(3)$ & $0.78(7)$ \\
& 150 & $3.3009(4)$ & $3.202(2)$ & $-3.00(6)$ \\
& 180 & $2.5672(3)$ & $2.393(2)$ & $-6.79(8)$ \\
\hline \multirow{3}{*}{1000} & 120 & $3.5817(6)$ & $3.607(3)$ & $0.71(8)$ \\
& 150 & $2.8635(4)$ & $2.776(2)$ & $-3.06(7)$ \\
& 180 & $2.3418(3)$ & $2.182(2)$ & $-6.82(8)$ \\
\hline \multirow{3}{*}{1200} & 120 & $3.0044(5)$ & $3.010(3)$ & $0.19(8)$ \\
& 150 & $2.4678(4)$ & $2.380(2)$ & $-3.56(8)$ \\
& 180 & $2.0770(3)$ & $1.926(2)$ & $-7.27(9)$ \\
\hline
\end{tabular}

Table 2: The numerical results of the tree-level, one-loop EW corrected cross sections and the relative EW radiative correction $\left(\delta_{e w} \equiv \Delta \sigma_{\text {tot }} / \sigma_{\text {tree }}\right)$ for the process $e^{+} e^{-} \rightarrow$ $H^{0} W^{+} W^{-}$, by taking $\sqrt{s}=400 \mathrm{GeV}, 600 \mathrm{GeV}, 800 \mathrm{GeV}, 1000 \mathrm{GeV}, 1200 \mathrm{GeV}$ and $m_{H}=120 \mathrm{GeV}, 150 \mathrm{GeV}, 180 \mathrm{GeV}$ separately. 

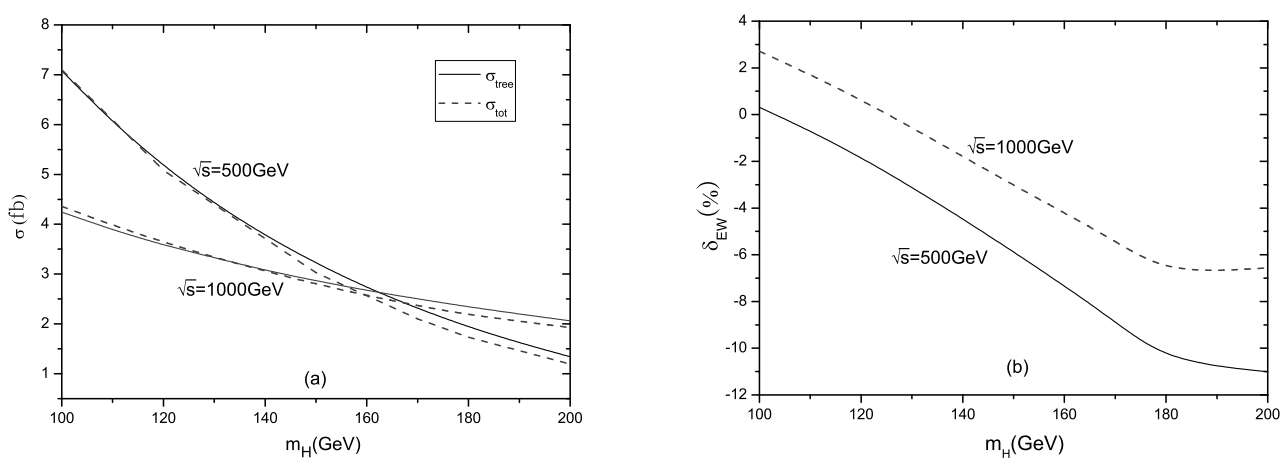

Figure 5: (a) The LO and EW one-loop corrected cross sections for the process $e^{+} e^{-} \rightarrow$ $H^{0} W^{+} W^{-}$as the functions of the Higgs-boson mass $m_{H}$ with $\sqrt{s}=500 \mathrm{GeV}, 1000 \mathrm{GeV}$. (b) The corresponding relative EW radiative corrections versus Higgs-boson mass.

for the process $e^{+} e^{-} \rightarrow H^{0} W^{+} W^{-}$as the functions of Higgs-boson mass $m_{H}$ by taking $\sqrt{s}=500 \mathrm{GeV}$ and $1000 \mathrm{GeV}$ separately, and their corresponding relative EW radiative corrections versus Higgs-boson mass are depicted in Fig [5(b). From these two figures we can see again the cross sections at LO and up to one-loop order for the process $e^{+} e^{-} \rightarrow H^{0} W^{+} W^{-}$are all sensitive to the Higgs-boson mass as already shown in Figs 4 (ab). And both curves for $\sqrt{s}=500 \mathrm{GeV}$ and $1000 \mathrm{GeV}$ in Fig.5(b) demonstrate that the absolute relative EW radiative corrections for $\sqrt{s}=500 \mathrm{GeV}$ and $1000 \mathrm{GeV}$ go up quickly, when Higgs-boson mass goes up from from $100 \mathrm{GeV}$ to $180 \mathrm{GeV}$, but become relative stable when $m_{H}$ runs from $180 \mathrm{GeV}$ to $200 \mathrm{GeV}$.

Since we consider the CP-conserving SM, the distributions of transverse momenta of $W^{-}$-boson should be the same as that of $p_{T}^{W^{+}}$. Therefore, we shall not provide the distribution of $p_{T}^{W^{-}}$but only the $p_{T}^{W^{+}}$. We depict the differential cross sections of transverse momentum of $H^{0}$-boson at the LO and up to NLO $\left(d \sigma_{L O, N L O} / d p_{T}^{H^{0}}\right)$ in Fig 5 (a), and the distributions of $d \sigma_{L O} / d p_{T}^{W^{+}}$and $d \sigma_{N L O} / d p_{T}^{W^{+}}$in Fig.5(b) separately, in the conditions of $m_{H}=120 \mathrm{GeV}$ and $\sqrt{s}=500 \mathrm{GeV}$. We can see from Figs 5 (a-b) that when the transverse momentum $p_{T}^{H^{0}}\left(p_{T}^{W^{+}}\right)$is in the range smaller than $90 \mathrm{GeV}$, the LO differential cross 

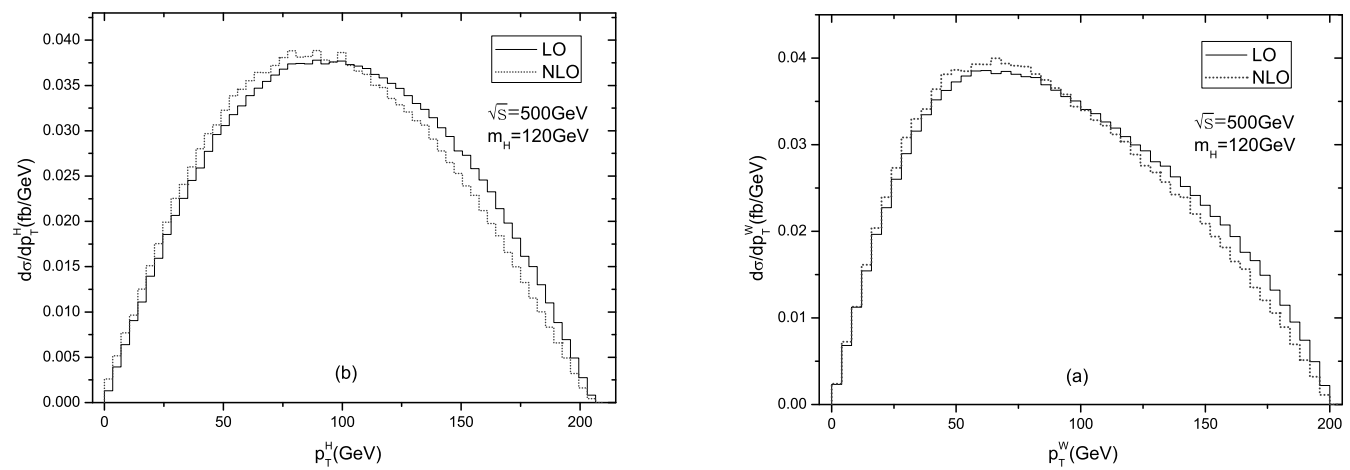

Figure 6: The distributions of the transverse momenta of $H^{0}$ - and $W^{+}$-boson for the $e^{+} e^{-} \rightarrow H^{0} W^{+} W^{-}$process at the LO and up to EW one-loop level with $\sqrt{s}=500 \mathrm{GeV}$ and $m_{H}=120 \mathrm{GeV}$. (a) for $H^{0}$-boson, (b) for $W^{+}$-boson.

section of $d \sigma_{L O} / d p_{T}^{H^{0}}\left(d \sigma_{L O} / d p_{T}^{W^{+}}\right)$is slightly enhanced by the EW one-loop corrections, while it is suppressed when $p_{T}^{H^{0}}\left(p_{T}^{W^{+}}\right)$is larger than $100 \mathrm{GeV}$.

We plot the distributions of $\mathrm{W}$-pair invariant mass, denoted as $M_{W W}$, at the LO and up to EW one-loop level in Fig.7 by taking $m_{H}=120 \mathrm{GeV}$ and $\sqrt{s}=500 \mathrm{GeV}$. We can see from the figure that when $M_{W W}<270 \mathrm{GeV}$ the one-loop EW corrections enhance the LO differential cross section $d \sigma_{L O} / d M_{W W}$ slightly, while in the $M_{W W}$ region being larger than $280 \mathrm{GeV}$ the $d \sigma_{L O} / d M_{W W}$ is obviously suppressed by the EW corrections.

\section{Summary}

The future $e^{+} e^{-}$linear collider, International Linear Collider(ILC), will probably offer the cleanest environment to probe the SM more precisely and discover the signature of new physics. In this paper we have shown that the phenomenological effects due to the contribution from complete one-loop EW terms of $\mathcal{O}\left(\alpha_{e w}^{4}\right)$, can be demonstrated in the study of the electron-positron into Higgs-boson in association with a W-boson pair in the standard model(SM) for all energies ranging from $300 \mathrm{GeV}$ to $1.2 \mathrm{TeV}$ at ILC. We 


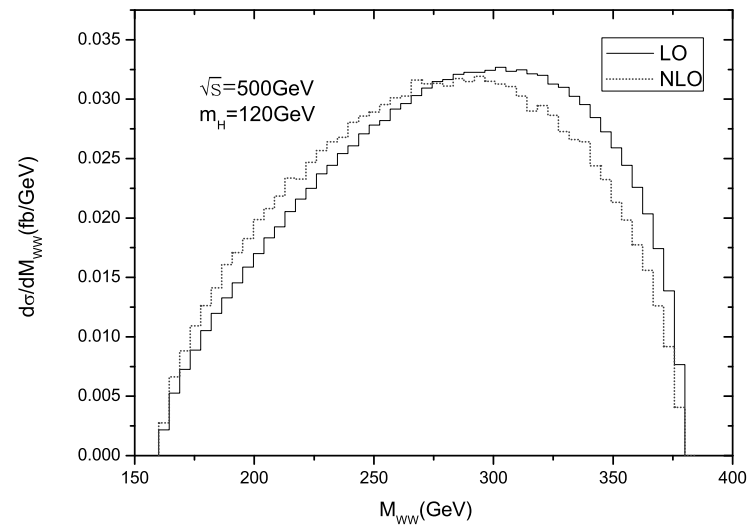

Figure 7: The distributions of the invariant mass of W-pair at the LO and up to EW one-loop level when $m_{H}=120 \mathrm{GeV}$ and $\sqrt{s}=500 \mathrm{GeV}$.

discuss the dependence of the effects coming from the EW NLO contribution to the cross section of process $e^{+} e^{-} \rightarrow H^{0} W^{+} W^{-}$, on colliding energy $\sqrt{s}$ and Higgs-boson mass. We present the LO and EW NLO corrected distributions of the transverse momenta of final particles and the differential cross section of $W$-pair invariant mass. We find that the LO cross section of process $e^{+} e^{-} \rightarrow H^{0} W^{+} W^{-}$is sensitive to Higgs-boson mass and generally suppressed by the one-loop EW corrections except when $m_{H}=120 \mathrm{GeV}$ and $\sqrt{s}>630 \mathrm{GeV}$. Our numerical results show that when $m_{H}=120 \mathrm{GeV}$ and $\sqrt{s}$ goes up from $300 \mathrm{GeV}$ to $1.2 \mathrm{TeV}$ the relative $\mathrm{EW}$ radiative correction varies from $-19.4 \%$ to $0.2 \%$. It shows that the EW one-loop radiative corrections to $e^{+} e^{-} \rightarrow H^{0} W^{+} W^{-}$process can be significant and should be included in any reliable analysis.

Acknowledgments: This work was supported in part by the National Natural Science Foundation of China, Specialized Research Fund for the Doctoral Program of Higher Education(SRFDP) and a special fund sponsored by Chinese Academy of Sciences. 


\section{References}

[1] S. L. Glashow, Nucl. Phys. 22, (1961) 579; S. Weinberg, Phys. Rev. Lett. 1, (1967) 1264; A. Salam, Proc. 8th Nobel Symposium Stockholm 1968, ed. N. Svartholm(Almquist and Wiksells, Stockholm 1968) p.367; H. D. Politzer, Phys. Rep. 14, (1974) 129.

[2] P. W. Higgs, Phys. Lett. 12, (1964) 132; Phys. Rev. Lett. 13, (1964) 508; Phys. Rev. 145, 1156 (1966); F. Englert and R. Brout, Phys. Rev. Lett. 13, 321 (1964); G. S. Guralnik, C. R. Hagen and T. W. B. Kibble, Phys. Rev. Lett. 13, (1964) 585; T. W. B. Kibble, Phys. Rev. 155, (1967) 1554.

[3] R.Barate et al., Phys. Lett. B565 (2003) 61.

[4] The LEP Collaborations ALEPH, DELPHI, L3, OPAL, and the LEP Electroweak Working Group. LEPEWWG/2007-01 and arXiv:0712.0929.

[5] Parameters for Linear Collider, http://www.fnal.gov/directorate/icfa/LC_parameters.pdf

[6] Proc. of the Workshop on " $e^{+} e^{-}$Collisions at $500 \mathrm{GeV}$. The Physics Potential", Munich, Annecy, Hamburg, Feb. 4 to Sept. 3, 1991, DESY publication, 92-123A/B, August 1992, ed. by P.M. Zerwas.

[7] Proc. of the Workshop on "Physics and Experiments with Linear Colliders", Saariselkä, Finland, 9-14 Sept., 1991, eds. R. Orawa, P. Eerola and M. Nordberg, World Scientific Publishing, Singapore (1992).

[8] K Abe et al., "Particle physics experiments at JLC", arXiv:hep-ph/0109166; J.A. Aguilar-Saavedra et al., TESLA Technical Design Report Pert III: Physics at an $e^{+} e^{-}$Linear Collider," arXiv:hep-ph/0106315; T Abe et al.," Linear collider physics resource book for Snowmass 2001. 2: Higgs and supersymmetry studies," in Proc. 
of the APS/DPF/DPB Summer Study on the Future of Particle Physics(Snowmass 2001) ed. R. Davidon and C. Quigg, arXiv:hep-ex/0106056.

[9] V Barger, T. Han and A. Stange, Phys. rev.D42 (1990) 777.

[10] M. Baillargeon, F. Boudjema, F.Cuypers, E. Gabrielli, and B. Mele, Nucl. Phys. B424 (1994) 343.

[11] Y.-J. Zhou, W.-G. Ma, R.-Y. Zhang, Y. Jiang, and L. Han, Phys. Rev. D73, 073009 (2006).

[12] T. Hahn, Comput. Phys. Commun. 140 (2001)418.

[13] T. Hahn, M. Perez-Victoria, Comput. Phys. Commun. 118 (1999)153.

[14] W.M. Yao, et al., J. of Phys. G33,1 (2006).

[15] G.'t Hooft and M. Veltman, Nucl. Phys. B153 (1979) 365.

[16] A. Denner, U Nierste and R Scharf, Nucl. Phys. B367 (1991) 637.

[17] G. Passarino and M. Veltman, Nucl. Phys. B160, 151 (1979).

[18] A. Denner and S. Dittmaier, Nucl. Phys. B658 (2003) 175.

[19] T. Ishikawa, et al., (MINMI-TATEYA collaboration) "GRACE User's manual version 2.0", August 1, 1994.

[20] A. Denner, Fortschr. Phys. 41, 307 (1993).

[21] B. W. Harris and J.F. Owens, Phys. Rev. D65 (2002) 094032, arXiv:hep-ph/0102128.

[22] G. 't Hooft and Veltman, Nucl. Phys. B153, 365 (1979). 